\title{
Développement d'une stratégie d'immunothérapie dans le traitement des cancers des voies aéro-digestives supérieures induits par des papillomavirus
}

\author{
Géraldine Lescaille, Fabien Pitoiset, Rodney Macedo, Christophe Huret, \\ Claude Baillou, Bertrand Bellier, François Lemoine
}

UPMC Université Paris 6 et UMR CNRS 7211/INSERM U959, Groupe hospitalier Pitié- Salpêtrière, 83 boulevard de l'Hôpital, 75013 Paris, France

\author{
geraldine.lescaille@gmail.com
}

L'alcool et le tabac sont les principaux facteurs de risque des cancers des voies aéro-digestives supérieures (VADS). L'implication des papillomavirus (HPV) dans certains cancers des VADS, principalement dans les cancers de l'oropharynx, a été récemment mise en évidence. On estime que 20 à 93\% des cancers de l'oropharynx (amygdale et base de langue) sont liés à l'HPV16 (Nasman et al. 2009, Chaturvedi et al. 2011), et ils expriment les oncoprotéines E6 et E7, comme dans la majeure partie des cancers ano-génitaux (Zur Hausen 1998). Des vaccins préventifs par pseudoparticules virales issus des protéines de la capside L1 et L2 des HPV, ont été développé contre les infections par l'HPV. Leur utilisation s'est révélée inefficace dans le traitement de tumeurs établies. Il apparait donc important de développer des stratégies adéquates pour contrôler les cancers induits par les HPV.

Dans cette étude, une stratégie de vaccination ADN permettant d'induire des pseudoparticules virales non infectieuses (VLPs), présentant l'antigène viral E7 de 1'HPV 16, a été développée. On parle ainsi de plasmo-rétro VLPs (pVLPs) du fait que les VLPs sont produites in vivo après l'administration d'un vecteur plasmidique contenant les séquences nécessaires et suffisantes à l'expression des protéines constitutives des VLPs, qui sont relarguées dans l'environnement extracellulaire. Les VLPs ainsi produites in vivo sont immunogènes et ont une meilleure immunogènicité que des protéines solubles. Cette stratégie vaccinale présente l'avantage d'une production simple, rapide, peu onéreuse et applicable à grande échelle.

Il a tout d'abord été montré ici que cette stratégie pouvait induire in vitro chez l'homme une réponse T CD8 spécifique d'E7 par technique ELIspot IFNg, à partir de cellules dendritiques issus de donneurs HLA-A2 et chargées avec des VLP E7 ; elles sont ensuite utilisées pour sensibiliser des lymphocytes T autologues. Chez la souris, la stratégie vaccinale pVLP-E7 a permis d'obtenir in vivo une protection préventive complète contre les tumeurs induites par l'HPV 16 (cellules TC-1). Dans un modèle curatif, une régression statistiquement significative des tumeurs a été observée lorsque les souris sont vaccinées précocement après l'induction des tumeurs. La capacité de cette stratégie à induire des réponses anti-tumorales a été également obtenue à des temps tardifs à condition d'associer des adjuvants à la vaccination $p V L P$. Ainsi, ce modèle pré-clinique montre que la stratégie plasmo-rétro VLP permet d'induire de bonnes réponses anti-tumorales dans un modèle murin de tumeur induite par les HPV 